\title{
Dependence of Resonance Energy Transfer on Exciton Dimensionality
}

\author{
Jan Junis Rindermann, Galia Pozina, Bo Monemar, Lars Hultman, \\ Hiroshi Amano and Pavlos G. Lagoudakis
}

\section{Linköping University Post Print}

N.B.: When citing this work, cite the original article.

Original Publication:

Jan Junis Rindermann, Galia Pozina, Bo Monemar, Lars Hultman, Hiroshi Amano and Pavlos G. Lagoudakis, Dependence of Resonance Energy Transfer on Exciton Dimensionality, 2011, Physical Review Letters, (107), 23, 236805.

http://dx.doi.org/10.1103/PhysRevLett.107.236805

Copyright: American Physical Society http://www.aps.org/

Postprint available at: Linköping University Electronic Press

http://urn.kb.se/resolve?urn=urn:nbn:se:liu:diva-73329 


\title{
Dependence of Resonance Energy Transfer on Exciton Dimensionality
}

\author{
Jan Junis Rindermann, ${ }^{1}$ Galia Pozina, ${ }^{2}$ Bo Monemar, ${ }^{2,3}$ Lars Hultman, ${ }^{2}$ Hiroshi Amano, ${ }^{4}$ and Pavlos G. Lagoudakis ${ }^{1, *}$ \\ ${ }^{1}$ School of Physics and Astronomy, University of Southampton, Southampton SO17 1BJ, United Kingdom \\ ${ }^{2}$ Department of Physics, Chemistry and Biology, Linköping University, Linköping S-581 83, Sweden \\ ${ }^{3}$ The Nanometer Structure Consortium, Lund University, Lund S-221 00, Sweden \\ ${ }^{4}$ Department of Electrical Engineering and Computer Science, Nagoya University, Chikusa-ku, Nagoya 464-8603, Japan
}

(Received 23 June 2011; published 30 November 2011)

\begin{abstract}
We investigate the dependence of resonance energy transfer from Wannier-Mott excitons to an organic overlayer on exciton dimensionality. We exploit the excitonic potential disorder in a single quantum well to tune the balance between localized and free excitons by scaling the Boltzmann distribution of excitons through temperature. Theoretical calculations predict the experimentally observed temperature dependence of resonance energy transfer and allow us to quantify the contribution of localized and free excitons. We show that free excitons can undergo resonance energy transfer with an order of magnitude higher rate compared to localized excitons, emphasizing the potential of hybrid optoelectronic devices utilizing resonance energy transfer as a means to overcome charge transfer related limitations.
\end{abstract}

DOI: 10.1103/PhysRevLett.107.236805

PACS numbers: 73.20.Mf, 78.47.jd

Hybrid optoelectronic devices, composed of different material classes such as epitaxially grown inorganic semiconductors and organic polymers, combine the superior electrical properties of inorganic crystals with the high photoluminescence yield across the visible spectrum that can be found in organic materials. For applications in light-emitting diodes, multicolor emission is desirable but challenging to achieve from epitaxially grown devices [1]. However, a blend of appropriately chosen fluorescent polymers, or quantum dots (QDs), yields multiple emission bands in the visible. They can be employed as highly efficient phosphors on UV or blueemitting quantum well (QW) light-emitting diodes based on III-nitride materials and offer an alternative to rare earth based phosphors, which are becoming increasingly scarce [2]. In such hybrid configurations, resonance energy transfer (RET) can overcome limitations for charge injection and transport in semiconducting polymers or QDs [3]. RET does not rely on carrier transport via electrical connectivity but on transfer of electron-hole pairs through dipolar coupling. In closely spaced configurations, RET can be orders of magnitude more efficient than radiative energy transfer [4]. Historically, this mechanism was first studied in the photosynthetic units in plant cells [5] before it was suggested as a pumping scheme in hybrid devices [6]. Theoretical work by Basko et al. predicted that the RET dynamics of an exciton in a QW is governed by the degree of localization, a parameter, which is unintentionally set by the growth conditions and purity of the materials used [7]. In this Letter, we observe and model the dependence of RET on temperature in a hybrid QW-organic configuration. We attribute an unexpected temperature dependence to the interplay between free and QD-like localized excitons in the QW and underline the effect of exciton localization on RET dynamics.

We study RET from a $L_{w}=2 \mathrm{~nm}$ thin single $\mathrm{Al}_{0.16} \mathrm{Ga}_{0.84} \mathrm{~N} / \mathrm{GaN} / \mathrm{Al}_{0.16} \mathrm{Ga}_{0.84} \mathrm{~N}$ QW with a $L_{b}=4 \mathrm{~nm}$ thin capping layer to an overlayer of the green emitting polyfluorene poly(9,9-dioctylfluorene-cobenzothiadiazole) (F8BT) end-capped with 2,5dimethylphenyl. Figure 1(a) shows the spectral overlap between the QW emission and the polyfluorene absorption. The thin capping layer enables nonradiative RET from the QW to the polyfluorene, which is inhibited in control samples with 6 and $9 \mathrm{~nm}$ thick capping layers. Figure 1(b) shows a schematic of the RET enabled structure. The QWs

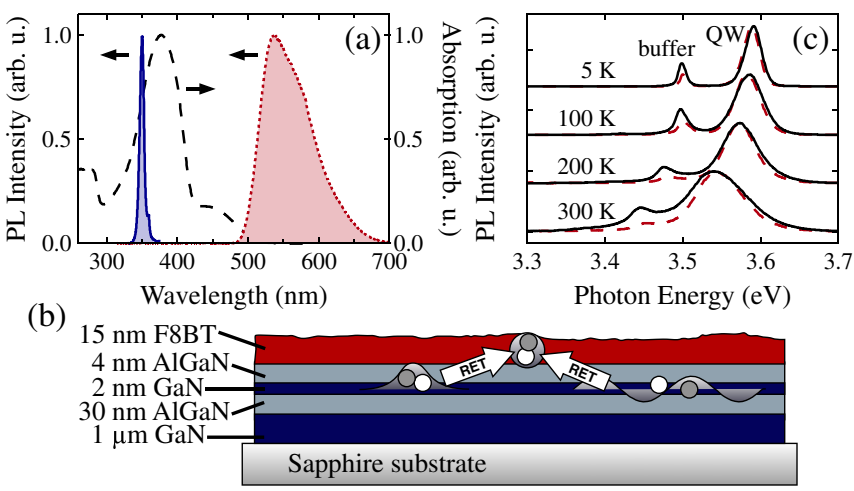

FIG. 1 (color online). Hybrid organic-inorganic configuration. (a) Spectral overlap between $\mathrm{QW}$ emission (blue line) and polyfluorene absorption (black dashed line). Red dotted line: polyfluorene emission. (b) Schematic of the hybrid configuration. (c) Spectral broadening and shift of QW emission with increasing temperature. Black solid line, without polyfluorene; red interrupted line, with polyfluorene. The emission spectrum shows a contribution from the $1 \mu \mathrm{m}$ thick GaN buffer layer. 
were grown on sapphire substrates by metal organic chemical vapor deposition using trimethyl gallium, trimethyl aluminum, and ammonia as precursors at a temperature of $1050^{\circ} \mathrm{C}$. The green emitting polyfluorene was spin-coated from a $20 \mathrm{mg} / \mathrm{ml}$ toluene solution to yield a $15 \mathrm{~nm}$ thin film as estimated by a contact profilometer measurement. RET from the QW is characterized in the time domain at various temperatures between $5 \mathrm{~K}$ and room temperature. For the measurement of time-resolved photoluminescence (PL), the QW and the polyfluorene were excited by the third harmonics from a Ti:sapphire femtosecond pulsed laser (energy density ca. $50 \mathrm{~W} \mathrm{~cm}^{-2}$ at $\lambda_{\mathrm{Ex}}=266 \mathrm{~nm}$ ) at a repetition rate of $75 \mathrm{MHz}$, and the emission was time- and energyresolved with a Hamamatsu syncroscan streak camera with $\sim 2$ ps resolution. Figure 1(c) shows the time integrated QW emission spectrum in the bare (QW only) and hybrid (QWpolyfluorene) configuration for different temperatures. At low temperatures, the linewidth is inhomogeneously broadened with a full width at half maximum (FWHM) of $25 \mathrm{meV}$ at $5 \mathrm{~K}$. In both the bare and hybrid configurations, the QW emission shows similar thermal broadening with increasing temperature leading to 90 and $71 \mathrm{meV}$ FWHM, respectively, at room temperature, and the emission energy shifts by $50 \mathrm{meV}$. The dynamics of RET is studied from the PL quenching of the QW exciton in the presence of the polyfluorene. The PL decay of the QW with and without the polyfluorene overlayer at different temperatures is shown in Fig. 2(a). At all temperatures [Fig. 2(a) shows only a subset of the data], the PL decay of the QW is accelerated in the presence of the polyfluorene. The QW PL decay is fully recovered upon removal of the polyfluorene layer. Figure 2(b) shows the extracted PL decay rates over a wide range of temperatures. The difference between the PL decay rates in the hybrid and bare configurations is particularly strong at temperatures between 100 and $170 \mathrm{~K}$. Charge transfer from the QW to the polyfluorene is prohibited due to the $4 \mathrm{~nm}$ thick undoped capping layer between the layers and, hence, cannot contribute to PL quenching. The emission from the QW control sample with the thicker capping layer $(6 \mathrm{~nm})$ remains unaltered after deposition of the polyfluorene layer, shown in the two lowermost graphs in Fig. 2(a). Therefore, the accelerated PL decay rate in the hybrid configuration can be attributed to RET mediated via near field dipolar coupling between the excitonic transition dipole moment and the polarizability of the organic layer. RET to the polyfluorene acts as an additional pumping source that can be identified on the rise time of the polyfluorene's PL dynamics [8]. Here, the polyfluorene is also directly optically excited by the laser pulse at $266 \mathrm{~nm}$ [see Fig. 1(a)]. A difference in the buildup of the polyfluorene's PL dynamics is evidenced in Fig. 2(e). Expectedly, a slower rise time is observed in the RET enabled configuration. Resonant back-transfer of energy from the F8BT to the QW does not occur because the typical excited state relaxation time in polyfluorene is shorter than
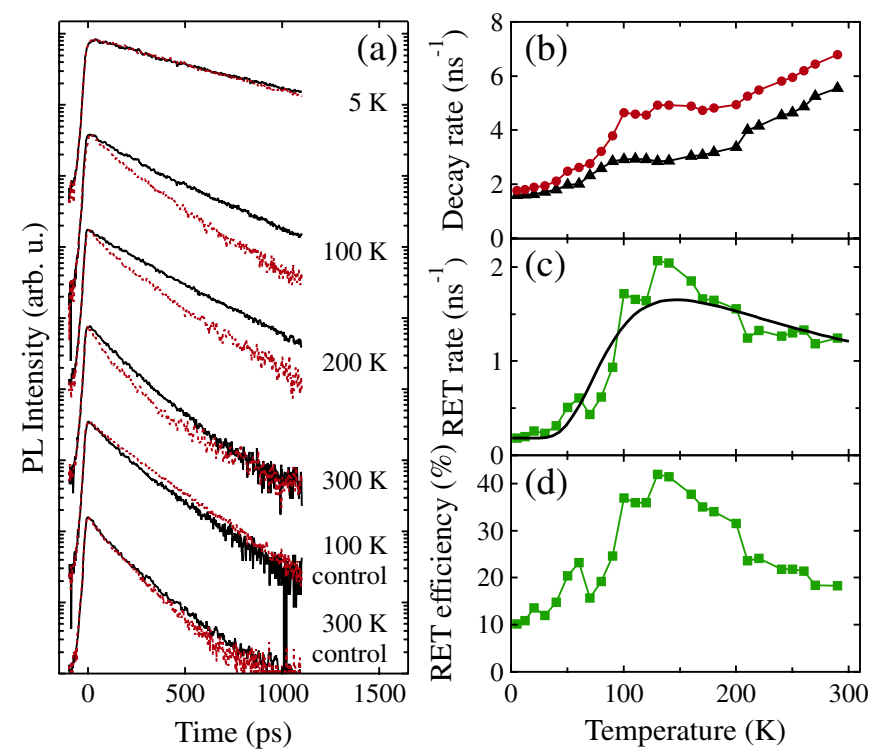

(e)

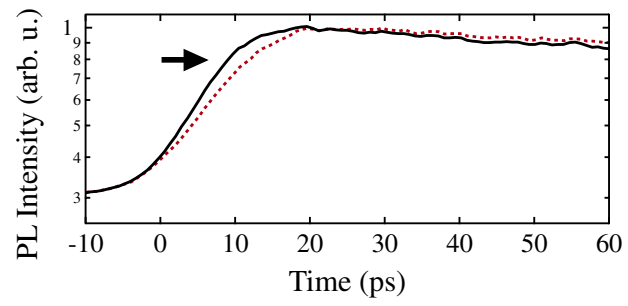

FIG. 2 (color online). Spectroscopic evidence of RET. (a) Photoluminescence decay of the QW before (black lines) and after (red lines) deposition of polyfluorene. The two lowermost graphs correspond to the control sample with a thicker capping layer. (b) Temperature dependence of exciton recombination in the QW before (black triangles) and after (red circles) deposition of polyfluorene. (c),(d) Energy transfer rate and efficiency vs temperature. The black line in (c) is the modeled temperature dependence. (e) Photoluminescence transient of the polyfluorene deposited on samples with a 4 (red dotted line) and $9 \mathrm{~nm}$ (black line) capping layer.

the characteristic transfer time [9]. This situation is referred to as weak coupling. The strong coupling regime in contrast leads to hybrid excitonic states when energy is coherently exchanged between the Wannier-Mott and Frenkel excitons [10].

The QW PL decay at different temperatures obeys an exponential law and can be characterized by a total decay rate $\Gamma_{\text {tot }}$, which can be written as the sum of the radiative decay rate and other, intrinsic to the $\mathrm{QW}$, nonradiative rates, excluding RET, $\Gamma_{\text {tot }}=\Gamma_{\text {rad }}+\Gamma_{\text {nonrad }}$. Therefore, the RET rate can be quantified from the increased decay rate in the presence of the polyfluorene epilayer, $\Gamma_{\mathrm{RET}}=\Gamma_{\text {tothybrid }}-$ $\Gamma_{\text {tot,bare }}$, where the subscripts hybrid and bare denote the QW with and without the polyfluorene epilayer, respectively. Finally, the efficiency of the process is defined as $\eta_{\mathrm{RET}}=\frac{\Gamma_{\mathrm{RET}}}{\Gamma_{\text {tothyrid }}}$. Figures 2(b)-2(d) show the temperature dependence of the total decay rate of the QW in the hybrid and bare configurations, the RET rate, and the RET 
efficiency. The total decay rate of the bare QW increases with temperature, due to the temperature dependence of other nonradiative recombination channels, intrinsic to the QW. These channels include carrier trapping and nonradiative recombination at defect states, such as dislocations or $\mathrm{AlGaN} / \mathrm{GaN}$ interfaces. This leads to a steep decrease of the quantum yield $Q Y_{\mathrm{QW}}$ from an assumed $100 \%$ at $5 \mathrm{~K}$ to below $30 \%$ at room temperature which was estimated from the PL decay rates. The rate of RET at different temperatures is shown in Fig. 2(c). It peaks around $130 \mathrm{~K}$, which is reproduced in the temperature dependence of the RET efficiency with a maximum efficiency of $43 \%$ [Fig. 2(d)]. A similar thermal behavior was reported for a hybrid QWQD configuration [11].

Förster's theory of RET considers Frenkel excitons that are bound to individual molecular sites and is temperature independent [5]. In contrast, in crystalline inorganic semiconductors the electronic excitations are propagating Wannier-Mott excitons characterized by a wave vector $\boldsymbol{k}$. With regard to the planar hybrid configuration considered here, it is known that the dipolar coupling between two planes separated by distance $d$ scales with

$$
V(k, d) \propto k e^{-k d},
$$

where $k$ is the magnitude of the relative in-plane wave vector $\boldsymbol{k}$ [7]. This nonmonotonic dependence of the dipolar coupling on the wave vector leads to a strong dependence of RET on exciton localization in real space. The pronounced temperature dependence of RET from excitons in disordered QWs can be understood from the wave vector dependence: With increasing temperature, a localized exciton becomes free and acquires kinetic energy. Whereas a free exciton is characterized by its in-plane wave vector, a localized exciton can be described by a distribution of wave vectors with a zero average.

Basko et al. calculated the rate of RET from localized and free QW excitons in the linear regime to a bulk epilayer of organic molecules [7] or QDs [12]. The rate of RET is calculated by applying Fermi's golden rule to the interaction of the electric field produced by the QW polarization with the polarizability of the polyfluorene. While the calculation takes into account the quantum mechanical wave function of the QW exciton, the properties of the organic molecules are summarized by the macroscopic complex dielectric function of the organic material. We model the experimentally observed temperature dependence of RET as due to a transfer of population from localized to free excitons with increasing thermal energy. Given the material parameters of the $\mathrm{QW}$, barrier, and organic layer, the rate of RET from free excitons depends strongly on their center of mass wave vector, which is determined by their kinetic energy. Similarly, RET from localized excitons depends on the localization length $L_{\mathrm{loc}}$, a measure for the width of the exciton wave function in real space. The corresponding rate of RET is then calculated by decomposing the exciton wave function in reciprocal space. Here, we simplify the description of the excitonic disorder in QWs and approximate the disorder potential using only two parameters: a characteristic $L_{\mathrm{loc}}$ for trapped excitons and a potential trap depth $V_{\text {trap }}$ which localized excitons have to overcome to propagate freely. These inplane potential traps are approximated with an isotropic harmonic potential. The harmonic approximation of the random potential is schematically shown in Fig. 3(a).

Following thermalization at a given temperature, the occupation of localized and free exciton states is determined by the Boltzmann factor and the density of states (DOS). Figure 3(b) depicts a schematic of the DOS. For free excitons, we consider a constant DOS above $V_{\text {trap }}$, corresponding to free excitonic states with the lowest quantum number in the QW. The contribution of localized exciton states to the DOS is approximated with a delta function at $E=0$. The relative area of the two parts of the DOS is a measure for the number of potential traps per area. Energy transfer from free carriers is not considered here because the excitation density is chosen sufficiently

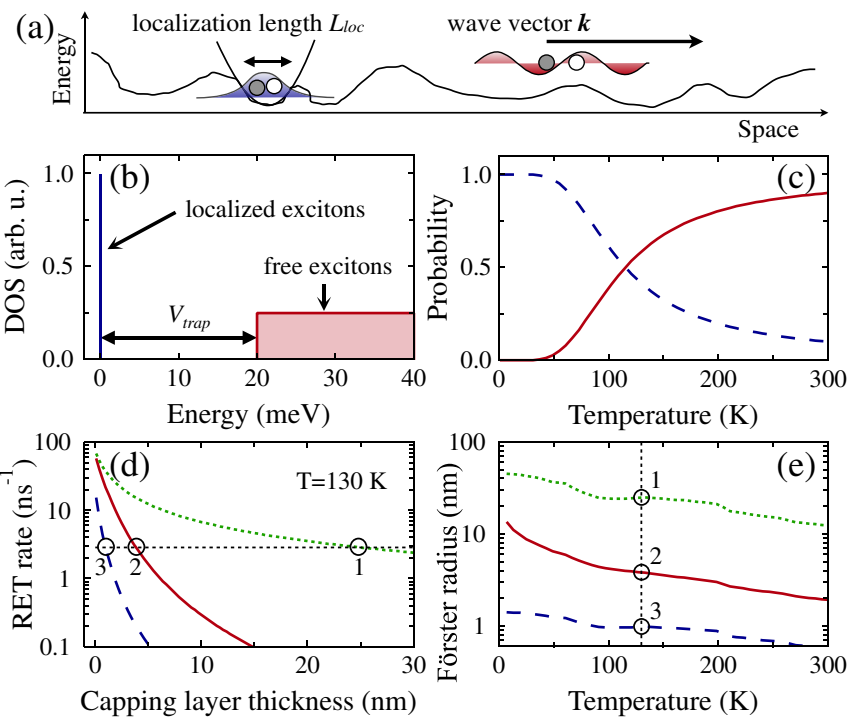

FIG. 3 (color online). Distribution of excitonic states in the imperfect QW. (a) Schematic of the random potential experienced by QW excitons and its harmonic approximation. (b) Density of states composed of a delta function for localized excitons and a flat contribution for free excitons in the QW (relative contributions not drawn to scale). (c) Population of localized (blue dashed line) and free (red line) excitons vs temperature. (d) Calculated RET rate for localized excitons (blue dashed line) and free excitons at $130 \mathrm{~K}$ (red line) for varying thickness of the capping layer. The dotted green line corresponds to the RET rate of the exciton with the optimum wave vector for each thickness. The thin horizontal line marks the exciton decay rate at $130 \mathrm{~K}$ without RET. (e) Temperature dependence of the Förster radii of localized (blue dashed line), free (red line), and excitons with the optimum wave vector (green dotted line). 
low, and thermalization of electrons and holes into excitons occurs much faster. The large exciton binding energy in $\mathrm{GaN}$ (23 meV in bulk and $92 \mathrm{meV}$ in infinitely thin QW) prohibits exciton dissociation at room temperature.

For the exciton ensemble, an average rate of RET at temperature $T$ can be defined as

$$
\left\langle\Gamma_{\mathrm{RET}}\right\rangle=\frac{1}{Z(T)} \int_{0}^{\infty} \Gamma_{\mathrm{RET}}(E) \operatorname{DOS}(E) e^{-\left(E / k_{B} T\right)} d E,
$$

where $Z(T)$ is the partition function, $k_{B}$ is Boltzmann's constant, and the integration is performed over energy. $\Gamma_{\mathrm{RET}}(E)$ is calculated for localized and free excitons using the formalism of Ref. [7]. As a result, excitons with wave vectors favorable for RET contribute substantially to the integral and dominate the $\left\langle\Gamma_{\mathrm{RET}}\right\rangle$ of the excitonic ensemble. The dipole moment of the excitonic transition is determined from the Kane energy $E_{0}=14 \mathrm{eV}$ [13] and temperature-dependent band gap $E_{g}=3.502 \mathrm{eV}-$ $0.79 \times 10^{-3} \frac{T^{2}}{T+1000 \mathrm{~K}} \mathrm{eV} / \mathrm{K}$ of wurtzite $\mathrm{GaN}$ through $\left|d^{v c}\right|^{2}=\frac{e^{2} \hbar^{2} E_{0}}{2 m_{0} E_{g}^{2}}$ and here is reduced by a factor of 0.63 to account for a reduced dipole moment in $\mathrm{GaN}$ due to the internal electric field in the heterostructure [14]. The exciton Bohr radius in the QW is approximated at $3 \mathrm{~nm}$ [15], and the translational mass of an exciton is taken as the sum of the effective masses of the electron $\left(m_{e}=0.2 m_{0}\right)$ and hole $\left(m_{h}=1.3 m_{0}\right)$, where $m_{0}$ is the free electron mass [13]. The dielectric constant of $\mathrm{GaN}$ is set to 9.5. The optical properties of the polyfluorene at the emission energy of the QW are approximated as isotropic with a macroscopic dielectric function $\epsilon_{350 \mathrm{~nm}}=2.9+0.5 i$. It has been reported that thin films of F8BT show birefringence due to alignment of the long molecules parallel to the surface [16]. This implies an anisotropic absorption, which is dominant at the $\pi-\pi^{*}$ transition but negligible for nonresonant excitation at $350 \mathrm{~nm}$. The absorption properties of the organic medium are virtually temperature independent. From the complete suppression of RET in the control sample with a $6 \mathrm{~nm}$ thin capping layer, we can consider the organic epilayer $(15 \mathrm{~nm})$ on top of the QW in our experiment sufficiently thick to be modeled with the infinitely thick polarizable epilayer approximation. We consider RET mediated via the excitonic dipole moment both in $(L)$ and out $(Z)$ of the QW plane. The corresponding rates differ at most by a factor of 3 , and here we take their average.

The measured temperature dependence of the RET rate in the exciton ensemble is modeled by adjusting the characteristic $L_{\mathrm{loc}}, V_{\text {trap }}$, and the relative contributions of localized and free excitons to the DOS. Figure 2(c) shows the theoretical calculation along with the measured temperature dependence of the RET rate. We find that the RET rate at low temperatures depends strongly on the characteristic localization length. A small value for the in-plane extension of the exciton wave function of $L_{\mathrm{loc}}=0.9 \mathrm{~nm}$ accounts for the measured RET rate of $0.23 \mathrm{~ns}^{-1}$ at low temperatures. For $\mathrm{GaN}$ with a lattice constant of $0.32 \mathrm{~nm}$, the localization length corresponds to approximately 9 lattice sites in the QW plane, which indicates that excitons are trapped in the potential disorder and not at individual impurity sites. The estimated value for $L_{\mathrm{loc}}$ is comparable to the average spacing between $\mathrm{Al}$ atoms in the barriers $(\sim 2 \mathrm{~nm})$ [15]. Only at elevated temperatures do excitons acquire adequate thermal energy to escape from potential traps and undergo RET with a rate depending on their wave vector. A trap depth of $20 \mathrm{meV}$ and an area density of potential traps of $4 \times 10^{11} \mathrm{~cm}^{-2}$ yield contributions from localized and free excitons to the RET rate that reproduce well the experimental data [see Fig. 2(c)]. Figure 3(c) shows the balance between localized and free excitons at different temperatures. For temperatures below $50 \mathrm{~K}$, the population of localized excitons remains nearly unchanged, which is reflected in the constant RET rate in this temperature range [see Fig. 2(c)]. The rate of RET then increases with temperature until about $130 \mathrm{~K}$, when free excitons dominate RET, but decreases again when the wave vector of free excitons becomes too large for efficient dipolar coupling. This behavior can be understood from the aforementioned dipolar coupling in a planar configuration [Eq. (1)].

The efficiency of RET to the polyfluorene film exceeds that of radiative pumping due to the steep decrease of $Q Y_{\mathrm{QW}}$ with increasing temperature. The efficiency $\eta_{\text {rad }}$ of radiative pumping is the product of the emission probability of a photon from the QW and absorption of the same photon by the organic overlayer; hence, $\eta_{\mathrm{rad}}=$ $Q Y_{\mathrm{QW}} P_{\mathrm{F} 8 \mathrm{BT}}$. The factor $P_{\mathrm{F} 8 \mathrm{BT}}$ considers isotropic emission from the QW, Fresnel reflection of the emitted light at the GaN/F8BT interface (3.6\%), and a thickness of $15 \mathrm{~nm}$ for the polyfluorene layer. In agreement with a previous study, the resulting efficiency for radiative energy transfer at room temperature is 2 orders of magnitude lower than the efficiency for nonradiative RET, $0.15 \%$ vs $22 \%$ [4], underlining the importance of RET for the efficient operation of hybrid devices.

The observed high efficiency of RET compared to radiative energy transfer is rather counterintuitive when considering the very stringent conditions of RET on the separation distance of the involved dipoles $\left(r^{-6}\right)$ described in Förster theory. The distance where the RET rate equals the sum of all other decay rates, the so-called Förster radius, is usually between 1 and $5 \mathrm{~nm}$ for molecular optical dipoles, rendering RET inefficient for larger separation distances. Here, we want to demonstrate that for Wannier-Mott excitons the Förster radius depends strongly on the excitons' dimensionality and can reach up to $25 \mathrm{~nm}$ in the studied configuration. We calculated the dependence of the RET rate on the thickness of the capping layer that separates the QW excitons and the polyfluorene overlayer. Figure 3(d) shows the calculated dependence for localized 
excitons, for free excitons at $130 \mathrm{~K}$, and for excitons with wave vectors that yield the optimum RET rate for each capping layer thickness. The Förster radii for the three cases are 1, 4, and $25 \mathrm{~nm}$, respectively. Figure 3(e) shows the temperature dependence of these values. It can be seen that the exciton with the optimum wave vector yields a Förster radius of $12 \mathrm{~nm}$ at room temperature. It becomes apparent that by appropriate engineering of the exciton dimensionality and in-plane wave vector the rate of RET can be dramatically increased by over an order of magnitude compared to pointlike localized excitons. Whereas at low temperatures RET is solely dependent on the exciton localization length $L_{\mathrm{loc}}$, the temperature that yields the highest number of excitons with the optimum wave vector is determined by the potential trap depth $V_{\text {trap }}$. High-purity materials and long annealing phases during QW growth yield a more homogenous and flatter QW potential landscape. An increased population of excitons with the optimum wave vector could also be achieved by a periodically modulated in-plane QW potential.

In summary, we have characterized qualitatively and quantitatively the explicit dependence of resonance energy transfer from QW excitons to an overlayer of organic molecules on the degree of exciton dimensionality. We vary the temperature of the exciton distribution as a means to tune the balance between localized and free excitons. A transition from localized to free excitons with increasing temperature gives rise to a maximum energy transfer efficiency of $43 \%$ at $135 \mathrm{~K}$ underlining the strong effect of exciton dimensionality on resonance energy transfer. The parameters that determine the observed temperature dependence are the characteristic exciton localization length $L_{\text {loc }}$ and trap depth $V_{\text {trap }}$ in the QW, as well as the trap density. In principle, these parameters can be tuned by adjusting the epitaxial growth conditions and optimized for efficient RET. We demonstrate that RET from free excitons to a polarizable medium obeys less stringent conditions compared to pointlike dipoles, allowing for RET rates up to an order of magnitude larger than the corresponding rates for localized excitons. These observations encourage further investigations in the optimization of RET enabled hybrid optoelectronic devices.

We acknowledge support by EPSRC through EP/ F013876/1 and EP/G063494/1 as well as FP7 through ITN-ICARUS and Network of Excellence, N4E. G. P. acknowledges support by the Swedish Governmental Agency for Innovation Systems (VINNOVA). L. H. acknowledges the Swedish Government Strategic Research Area Grant in Materials Science, AFM. J. J. R. is indebted to the ReinerLemoine-Stiftung, Germany for financial support.
Note added in proof.-Recently, we became aware of an excellent review on hybrid organic-inorganic nanostructures for optoelectronic applications [17].

*pavlos.lagoudakis@soton.ac.uk

[1] E.F. Schubert, Light-Emitting Diodes (Cambridge University Press, Cambridge, England, 2006).

[2] C. R. Belton, G. Itskos, G. Heliotis, P. N. Stavrinou, P. G. Lagoudakis, J. Lupton, S. Pereira, E. Gu, C. Griffin, B. Guilhabert, I. M. Watson, A. R. Mackintosh, R. A. Pethrick, J. Feldmann, R. Murray, M. D. Dawson, and D. D. C. Bradley, J. Phys. D 41, 094006 (2008); G. Itskos, C. R. Belton, G. Heliotis, I. M. Watson, M. D. Dawson, R. Murray, and D.D.C. Bradley, Nanotechnology 20, 275207 (2009).

[3] S. Chanyawadee, P. Lagoudakis, R. Harley, M. Charlton, D. Talapin, H. Huang, and C. Lin, Adv. Mater. 22, 602 (2010).

[4] G. Itskos, G. Heliotis, P. G. Lagoudakis, J. Lupton, N. P. Barradas, E. Alves, S. Pereira, I. M. Watson, M.D. Dawson, J. Feldmann, R. Murray, and D. D. C. Bradley, Phys. Rev. B 76, 035344 (2007).

[5] T. Förster, Naturwissenschaften 33, 166 (1946).

[6] D. Dexter, J. Lumin. 18-19, 779 (1979).

[7] D. M. Basko, G. La Rocca, F. Bassani, and V. Agranovich, Eur. Phys. J. B 8, 353 (1999).

[8] S. Chanyawadee, P. G. Lagoudakis, R. T. Harley, D. G. Lidzey, and M. Henini, Phys. Rev. B 77, 193402 (2008); S. Chanyawadee, R. T. Harley, M. Henini, D. V. Talapin, and P. G. Lagoudakis, Phys. Rev. Lett. 102, 077402 (2009).

[9] M. Ariu, M. Sims, M. D. Rahn, J. Hill, A. M. Fox, D. G. Lidzey, M. Oda, J. Cabanillas-Gonzalez, and D. D.C. Bradley, Phys. Rev. B 67, 195333 (2003).

[10] V. M. Agranovich, G. C. La Rocca, F. Bassani, H. Benisty, and C. Weisbuch, Opt. Mater. 9, 430 (1998).

[11] S. Rohrmoser, J. Baldauf, R. T. Harley, P. G. Lagoudakis, S. Sapra, A. Eychmüller, and I. M. Watson, Appl. Phys. Lett. 91, 092126 (2007).

[12] D. M. Basko, G.C. La Rocca, F. Bassani, and V.M. Agranovich, Phys. Rev. B 71, 165330 (2005).

[13] I. Vurgaftman, J. Meyer, and L. Ram-Mohan, J. Appl. Phys. 89, 5815 (2001).

[14] G. Traetta, R. Cingolani, A. Di Carlo, F. Della Sala, and P. Lugli, Appl. Phys. Lett. 76, 1042 (2000).

[15] M. Gallart, A. Morel, T. Taliercio, P. Lefebvre, B. Gil, J. Allegre, H. Mathieu, N. Grandjean, M. Leroux, and J. Massies, Phys. Status Solidi A 180, 127 (2000).

[16] C. M. Ramsdale and N. C. Greenham, J. Phys. D 36, L29 (2003).

[17] V.M. Agranovich, Y.N. Gartstein, and M. Litinskaya, Chem. Rev. 111, 5179 (2011). 